\title{
Dual behavior of caustic optical beams facing obstacles
}

\author{
Pablo Vaveliuk,,${ }^{1, *}$ Óscar Martínez-Matos, ${ }^{2}$ Yu-Xuan Ren, ${ }^{3}$ and Rong-De $\mathrm{Lu}^{3}$ \\ ${ }^{1}$ Centro de Investigaciones Ópticas (CONICET La Plata - CIC), Camino Centenario y 506, P.O. Box 3, 1897 Gonnet, Argentina \\ ${ }^{2}$ Departamento de Óptica, Facultad de Ciencias Físicas, Universidad Complutense de Madrid, \\ Avenida Complutense s/n, 28040 Madrid, Spain \\ ${ }^{3}$ Physics Experiment Teaching Center, University of Science and Technology of China, Hefei 230026, China
}

(Received 28 March 2017; published 23 June 2017)

\begin{abstract}
A full propagation analysis on both fold-type and cusp-type caustic optical beams under various setups of obstructions is theoretically and experimentally performed. It is demonstrated that the self-healing property of caustic optical beams that include the famous Airy beam is a quite relative property. In fact, fold-type and cusp-type beams cannot only behave as self-healing beams by blocking the main intensity peak, but also behave as self-breaking ones in a nonintuitive manner: by blocking a lateral side of the beam without touching the central intensity peak. The regeneration and rupture processes of caustic beams follow a nonlocal propagation dynamic unlike the other conventional beams. Moreover, deep differences between fold and cusp caustic beams are pointed out once facing certain obstructions. The cusp-caustic beam can be broken down by the obstacle placed in a dark zone outside the caustic region, while the fold-type one remains unaltered. This beam rupture confirms the key role of a hidden propagating field in the shadow region for cusp beams that coexist with the evanescent one. The obtained results cast down the established idea that the Airy beam is a robust self-healing beam since any caustic beam can behave in a dual manner depending on the obstruction location. These facts open up different perspectives for the applications in which the self-healing properties of the beam are relevant.
\end{abstract}

DOI: 10.1103/PhysRevA.95.063838

\section{INTRODUCTION}

An optical beam is self-healing if its transverse intensity profile is barely affected, after propagation, by a small blocking in amplitude and/or phase placed in its trajectory. One of the reasons for a wide and growing interest in the famous accelerating Airy Beams (AiB) $[1,2]$ and other curved caustic beams [3] is their reported ability to self-heal during propagation [3-8]. The applications of this feature in optical micromanipulation and correlated branches [9] and in beam propagation through inhomogeneous and turbulent media $[10,11]$ encouraged the crescent study of this class of beams. The self-healing property of Airy beams basically consists in the "regeneration" of the main curved lobe when it is obstructed. To give a physical interpretation of this feature, several viewpoints were considered such as, for instance, the evolution of the Poynting vector [4], the evolution of the beam phase [8], and an analysis based on exact solutions of the wave equation [5,12]. The analysis done in Ref. [13] states that the main features of Airy beams, i.e., weak diffraction, curved trajectories, and self-healing, are a consequence of a nonlocal wave dynamics produced by a sideways ray contribution to the field. There is no explicit analysis of the beam behavior facing real obstacles in that work such that the predictions for this effect were indirectly induced from the analysis on the other properties of the Airy beam. There is, besides, another key matter related to the caustic structure of a curved beam that should affect its self-healing behavior: the symmetry of the spectral phase [14]. The odd symmetry gives rise to a fold-type caustic optical beam, while the even symmetry gives rise to a cusp-type one. The more studied fold caustic beam is the

*pablov@ ciop.unlp.edu.ar
AiB [1,2] while the Pearcey beam [3] and the symmetric Airy beam (SAB) [15-17] are examples of cusp-caustic beams. In fact, both caustic structures comprise an infinite number of beams with different curvatures depending on the value of the phase power that can be any real value [14]. Surely, the beams belonging to each caustic structure should behave in a different fashion facing identical setups of obstructions. However, there does not exist an overall analysis that spans caustic beams facing different setups of obstructions pointing out, in addition, the role of the spectral phase symmetry.

From the above-mentioned facts, this paper is devoted to perform a robust propagation analysis on fold-type and cusptype caustic beams facing different setups of obstructions. The theoretical analysis is performed by using two complementary approaches: the geometric one arising from the catastrophe theory on caustic structures and the wave-field analysis based on integral representations of the propagation field. Moreover, an experimental analysis is carried out in order to support the theoretical predictions. The findings of this work can be extended to other reported caustic beams with arbitrary complex trajectories [18-20] and other types of selfbending and self-healing beams such as Mathieu and Weber beams [21].

\section{THEORETICAL FOUNDATION}

\section{A. Geometric approach from catastrophe theory}

The Caustic-Catastrophe theory [22-24] gives a geometrical description of the ray scheme responsible for the caustic curve formation. Assume a two-dimensional (2D) curved beam propagating in free space in the $z$ direction that is partially blocked by one obstacle placed along the transverse direction $x$. The starting point is the full phase of the beam angular 
spectrum of power $n$ and symmetry $q[14]$,

$$
\Phi_{n}^{(q)}(K ; s, \xi)=C_{n} \operatorname{sgn}^{q}(K)|K|^{n}+\xi\left(k^{2}-\frac{K^{2}}{2}\right)+s K,
$$

with $K$ the dimensionless spatial frequency that is the conjugate variable of the normalized transverse spatial coordinate $s=x / x_{0}$, where $x_{0}$ is a transverse scale characterizing the beam. The normalized propagation coordinate is $\xi=z \lambda /\left(2 \pi x_{0}^{2}\right)$, and $k=(2 \pi / \lambda) x_{0}$ is the normalized freespace wave number with $\lambda$ being the free-space wavelength. The normalization constant $C_{n}$ guarantees the same range of phase variation for a given range of $K$. Here, $\operatorname{sgn}(\cdot)$ is the sign function and $|\cdot|$ is the absolute value. Under the catastrophe theory framework, $\Phi_{n}^{(q)}$ is called the generating function in which the spatial frequency $K$ plays the role of internal or state variable and the spatial variables $(s, \xi)$ act as external or control parameters $[22,23]$. The generating function is linear in terms of the control parameters and composed of three terms. The first one, $C_{n} \operatorname{sgn}^{q}(K)|K|^{n}$, is the germ of the generating function and does not depend on the control parameters. This term plays a key role since it governs the dynamics of the caustic structure of the beam. The other two unfolding terms, depending on control variables, are the paraxial propagator term and the Fourier transform term. The ray pattern associated to the beam, and thereby its caustic structure, can be obtained by differentiating the generating function with respect to the internal variable and setting the derivative to zero [22,23],

$$
\frac{\partial \Phi_{n}^{(q)}}{\partial K}=n C_{n} \operatorname{sgn}^{q+1}(K)|K|^{n-1}-\xi K+s=0 .
$$

Equation (2) evaluates the stationary points, say $K_{\text {st }}$, so that each one represents a particular family of rays with linear trajectory in the $s \xi$ plane. The number of solutions $K_{\text {st }}$ of (2) then coincides with the number of rays intersecting at a given point $(s, \xi)$. The watermark of catastrophe optics lies in that several rays can pass through the same point, which allows one to have an "envelope," which is precisely the caustic curve. On a caustic, two or more rays acquire the same direction, becoming parallel [23]. In practice, a "ray annihilation" occurs on the caustics with a jumpwise variation of the number of rays passing through the caustic curve giving rise to a catastrophe. The equations representing the caustic curves are obtained by setting to zero the second-order partial derivatives of $\Phi_{n}^{(q)}$,

$$
\frac{\partial^{2} \Phi_{n}^{(q)}}{\partial K^{2}}=n(n-1) C_{n} \operatorname{sgn}^{q}(K)|K|^{n-2}-\xi=0 .
$$

From a geometric viewpoint, Eq. (3) indicates that the projection of the ray surface onto the $s \xi$ space gives rise to singularities or catastrophes identified as caustics. Thereby, in order to obtain the equation of the caustic curve, the value of $K$ obtained from (3) must be replaced into (2). Explicitly, this caustic curve equation can be written as [14]

$$
\operatorname{sgn}^{q}(s)|s|+A_{n} \operatorname{sgn}^{q+1}(\xi)|\xi|^{\frac{n-1}{n-2}}=0,
$$

where

$$
A_{n}=C_{n}^{1 /(2-n)}(2-n) n^{1 /(2-n)} /(n-1)^{\frac{n-1}{n-2}} .
$$

The blockade of a subset of rays by an obstacle breaks down a part of the caustic curve. The caustic rupture extends between the spatial region limited by the rays touching the edges of the obstacle, say the "blocking limit rays." If the obstacle is placed at the plane $\xi=\xi_{0}$ in the interval $s_{0 a}<s<s_{0 b}$, the blocking limit rays are determined from the fixed values $K_{0 a}, K_{0 b}$ that are given by the algebraic equations

$$
n C_{n} \operatorname{sgn}^{q+1}\left(K_{i}\right)\left|K_{i}\right|^{n-1}-\xi_{0} K_{i}+s_{i}=0,
$$

with $i=0 a, 0 b$. This picture fully predicts the region and spatial extension of the caustic curve rupture as a function of the position and length of the obstacle.

\section{B. Wave field from an integral representation}

The undulatory description of the wave field up to the obstacle plane, say $\xi=\xi_{0}$, is performed from the angular spectrum formalism [25,26]. The dimensionless wave field $u$, solution of paraxial equation $2 i \partial_{\xi} u+\partial_{s s} u=0$, is calculated by means of the integral equation,

$$
u_{n}^{(q)}(s, \xi)=\frac{1}{2 \pi} \int_{-\infty}^{+\infty} U_{n}^{q}(K) e^{i\left[\xi\left(k^{2}-\frac{K^{2}}{2}\right)+s K\right]} d K
$$

where

$$
U_{n}^{q}(K)=V(K) e^{i C_{n} \operatorname{sgn}^{q}(K)|K|^{n}}
$$

is the complex amplitude of the angular spectrum with $V$ being the real amplitude. $U$ can be viewed as the "beam's signature" since it defines the beam structure and dynamics in propagation [15]. The wave field at $\xi=\xi_{0}$, say $u=u_{0}$, is established from Eq. (6). Notice that the indices $n$ and $q$ in the wave field are omitted for simplicity. The obstruction is mathematically accounted at the plane $\xi=\xi_{0}$ as

$$
u_{0}^{\dagger}\left(s_{0}\right)=u_{0}\left(s_{0}\right) h\left(s_{0}\right)
$$

with $s_{0}$ the transverse coordinate on this plane and

$$
h\left(s_{0}\right)= \begin{cases}0 & \text { if } s_{0 a} \leqslant s_{0} \leqslant s_{0 b} \\ 1 & \text { otherwise }\end{cases}
$$

The obstacle cancels out the field in the interval $\left[s_{0 a}, s_{0 b}\right]$. The wave field $u_{0}^{\dagger}\left(s_{0}\right)$ is the input field of the Huygens-Fresnel integral [27],

$$
u_{F}(s, \xi)=\frac{e^{i\left(2 \pi x_{0} / \lambda\right)^{2} \xi}}{i \sqrt{2 \pi \xi} x_{0} / \lambda} \int_{-\infty}^{+\infty} u_{0}^{\dagger}\left(s_{0}\right) e^{-i\left(s-s_{0}\right)^{2} /(2 \xi)} d s_{0},
$$

from which the output field $u_{F}$ is calculated at any output plane $\xi>\xi_{0}$. Equations (1)-(9) provide the theoretical foundation to quantitatively determinate both the ray pattern and the intensity distribution of the wave field in the $\xi s$ space facing arbitrary obstructions. Thereby, a rigorous analysis can be done by considering several setups of obstacles in addition to the phase symmetry of the beam angular spectrum that characterizes fold-type and cusp-type caustic beams.

\section{THEORETICAL RESULTS}

In the following, we perform the simulations for the particular value $n=3$. The value $q=1$ leads to an antisymmetric spectral phase and $q=0$ leads to a symmetric one. Thereby, 

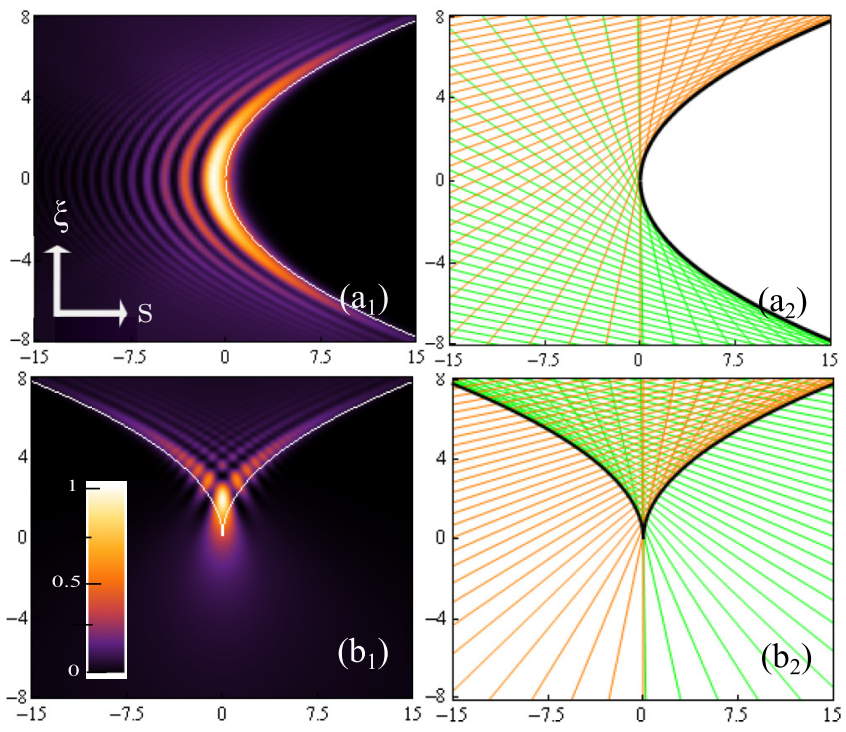

FIG. 1. $\left(\mathrm{a}_{1}\right)$ Intensity distribution normalized to its maximum value, $I / I_{\max }$, vs spatial coordinates $s$ and $\xi$ for $\mathrm{AB}$. The intensity scale $[0,1]$ corresponds to $\left[0, I / I_{\max }\right] .\left(\mathrm{a}_{2}\right)$ Spatial ray distribution for $\mathrm{AB}$ [orange rays (those coming from the left) for $K>0$ and green rays (those coming from the right) for $K<0]$. $\left(b_{1}\right),\left(b_{2}\right)$ Same, but for $\mathrm{SAB}$. White and black curves in the undulatory and geometric representations, respectively, are the caustic curves that are tangent to each of the rays in one point.

the values $n=3$ and $q=1$ determine the accelerating Airy beam [1] that is, undoubtedly, the more famous fold-type caustic beam, while the values $n=3$ and $q=0$ determine the recently generated SAB [15]. All the theoretical and experimental results obtained for these particular beams can be extended for any real value of $n$ such that they are valid for the overall fold-type and cusp-type beams as demonstrated in [14]. Figure 1 shows the spatial distribution of the light intensity from Eqs. (6)-(9) and the ray pattern from Eqs. (2)-(5) for (a) $\mathrm{AB}$ (fold-type caustic) and (b) SAB (cusp-type caustic) without obstructions. A fold-type caustic arises from the intersection of two rays inside the caustic region generating a fringe-type interference (the secondary lobes), and zero rays outside this region. Only evanescent waves exist beyond the caustic curve. In return, cusp-type caustic arises from the intersection of three rays inside the caustic region generating a dot-type interference, and one ray outside this region. This indicates the coexistence between evanescent and propagating waves beyond the caustic curve. The quite different ray morphology for both types of beams must yield a different propagation dynamics in the presence of identical obstacles.

We first begin by blocking the main intensity lobe of the $\mathrm{AB}$ and $\mathrm{SAB}$ with an obstacle placed at the beam center on $\xi=0$. The wave-field simulations are visualized in Fig. 2( $\left.a_{1}\right)$ for $A B$ and Fig. 2( $\left.b_{1}\right)$ for SAB. It is observed that the curved main lobe of $\mathrm{AB}$ is not broken by the obstacle, except at its neighborhood, confirming its self-healing feature. Our simulations provide evidence that the $\mathrm{SAB}$ also presents a self-healing characteristic supporting preliminary results obtained in [28].
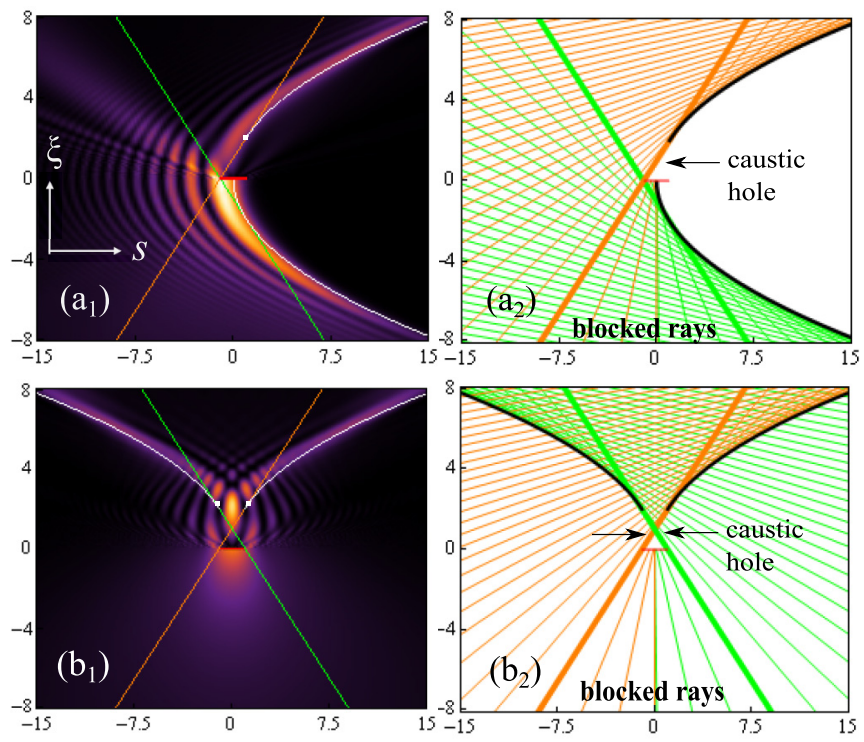

FIG. 2. $I / I_{\max }$ and ray distribution vs $(s, \xi)$ in the presence of an obstacle placed at the Fourier plane $(\xi=0)$ partially blocking the main lobe in the interval $-1 \leqslant s \leqslant 1$, (a) for $\mathrm{AB}$ and (b) for $\mathrm{SAB}$. Notice that the blocking limit rays $\left[\left(\mathrm{a}_{1}\right),\left(\mathrm{b}_{1}\right)\right.$ (orange and green) thin lines and $\left(a_{2}\right),\left(b_{2}\right)$ (orange and green) thick lines] delimit the caustic curve cut off by the obstacle [(white and black) curved lines in undulatory and geometric configurations, respectively].

In fact, notice that the main symmetric specular lobes are not affected by the perturbation except at its neighborhood. A robust explanation of this phenomenon is tackled from the full ray map taken into account for the rays blocked by the obstacle. The results are visualized in Fig. $2\left(\mathrm{a}_{2}\right)$ for $A B$ and Fig. 2( $\left.b_{2}\right)$ for SAB. Notice that the blocked rays are those that would form the caustic curve at the neighborhood to $\xi=0$. Hence, the caustic breaking is then only produced in this region that is delimited by the "blocking limit rays." The rest of the caustic curve remains unaltered. The geometric setup in Figs. $2\left(a_{2}\right)$ and $2\left(b_{2}\right)$ exemplifies the self-healing effect in fold-type and cusp-type beams. It is clear that the curved main lobes do not follow a local self-dynamics in the sense that this central lobe builds itself during propagation. On the contrary, one could assert, strictly speaking, that the main curved lobes are formed by a nonlocal contribution of rays coming from sideways regions far away from it that are not blocked by the obstacle. In summary, the obstacle placed at the beam center affects neither the structure of the fold-type caustic beam nor the structure of the cusp-type caustic beam except at the obstacle neighborhood.

In the above case, the nonlocal dynamics plays a "constructive role" in the beam regeneration process. However, the nonlocal dynamics can also play a "negative role" in the sense that the central lobe can be broken down by blocking a lateral side of the beam. This peculiar effect has not been tackled in the literature. Figure 3 illustrates this framework in which the obstacle is located at the left side of the beam center. The spatial distribution of the light intensity is shown in Fig. 3( $\left.a_{1}\right)$ for $\mathrm{AB}$ and Fig. 3(b $\left.\mathrm{b}_{1}\right)$ for SAB. Clearly, the obstacle blocks the main intensity lobe on $\xi=0$ neither for $\mathrm{AB}$ nor for $\mathrm{SAB}$. In spite of this, it is observed that the beam main lobe bending 

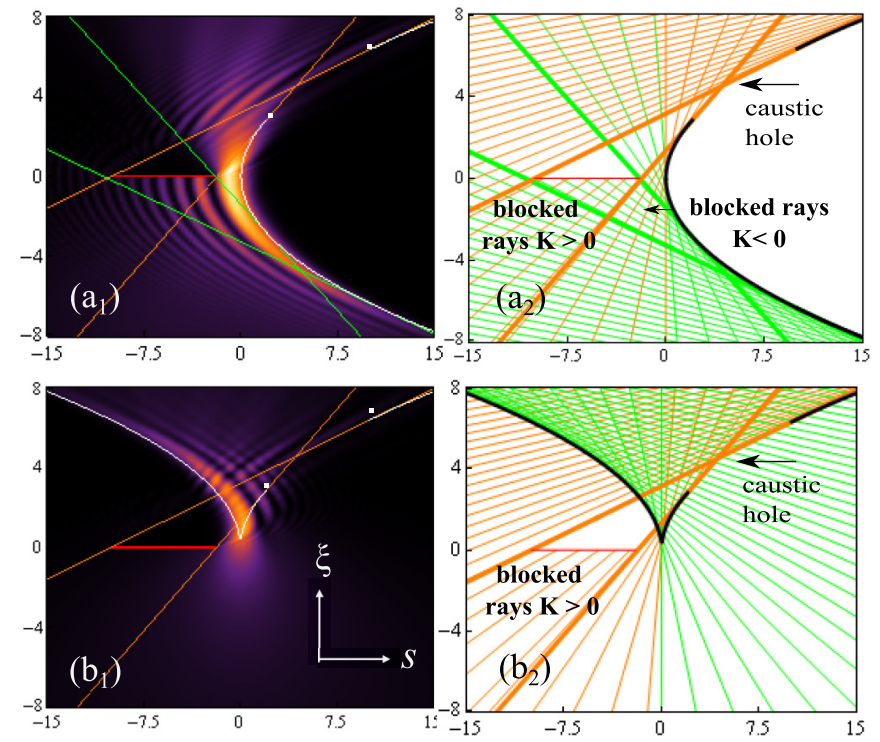

FIG. 3. $I / I_{\max }$ and ray distribution vs $(s, \xi)$ in the presence of an obstacle placed at the Fourier plane $(\xi=0)$ blocking the left lateral sideways of the beam in the interval $-10 \leqslant s \leqslant-2$, (a) for $\mathrm{AB}$ and (b) for SAB. Unlike Fig. 2, only the blocking limit rays for $K>0$ delimit the caustic curves cut off by the obstacle [those coming from the left side going to the right side represented by $\left(a_{1}\right),\left(b_{1}\right)$ (orange) thin lines and $\left(a_{2}\right),\left(b_{2}\right)$ (orange) thick lines].

to the opposite sideways to the obstacle breaks down during propagation. For AB [Fig. 3( $\left.\mathrm{a}_{1}\right)$ ], the blockade of secondary lobes to the left sideways of the main one results in a full destruction, almost immediately behind the obstacle plane, of the unique main lobe curved to the right side. For SAB [Fig. 3( $\left.b_{1}\right)$ ], the lateral obstruction breaks down the right-side lobe after the obstacle plane, leaving the left one invariant.

How does this peculiar phenomenon occur? Again, the ray scheme in the presence of the obstruction explains this feature. Notice from Figs. 3( $\left.a_{2}\right)$ and $3\left(b_{2}\right)$ how the nonlocal dynamics in propagation is responsible for such a peculiar behavior for both type of beams. For AB [Fig. 3( $\left.a_{2}\right)$ ], the rays proceeding from the left side contribute to the formation of the right side of the fold caustic curve. By blocking a part of them, as Fig. 3( $\left.a_{2}\right)$ shows, it inhibits the making of a part of the caustic curve. Notice that the blocking limit rays delimit the "caustic hole" and therefore determine the length of this. The rays proceeding from the right side (green straight lines) form the caustic curve for $\xi>0$. The rays proceeding from the right side do not participate in the caustic rupture. They only break down a part of the secondary lobes delimited by the blocking limit rays. After the caustic hole, the caustic curve reemerges from the unblocked rays and thereby the beam should re-self-heal. This is visualized from Figs. 3( $\left.a_{1}\right)$ and $3\left(\mathrm{a}_{2}\right)$ where the reemerging of the caustic curve from $\xi \gtrsim 6$ is clear. However, at this propagation stage, the beam already presents a strong spreading such that the main intensity lobe accompanying the caustics is not appreciated due to its negligible energy. For practical effects, the main lobe is fully broken down. For the SAB [Fig. 3( $\left.b_{2}\right)$ ], the process of the caustic rupture by the obstacle is equivalent. The equivalent behavior is because the left ray pattern has identical structure for both caustic beams. Hence, the caustic hole is equivalent for both structures, as shown in Figs. $3\left(a_{2}\right)$ and $3\left(b_{2}\right)$. For SAB, like $A B$, the rays arising from the right side (green straight lines) neither participate in the caustic breaking, nor are even blocked by the obstacle. In spite of this similarity, there is an essential difference related to the fact that the obstacle was placed inside the caustic region for $A B$ and outside this for SAB.

Notice from Figs. 3( $\left.a_{1}\right)$ and $3\left(b_{1}\right)$ that the obstacle in the $\mathrm{SAB}$ case lies in a dark region, unlike the $\mathrm{AB}$ where secondary curved lobes exist. The outside region to the cusp-caustic curve possessing one ray in each point in the region indicates the existence of a propagation wave field, coexisting with the evanescent one. This imperceptible propagating field is directly responsible for the building of the opposite main lobe. This is a clear manifestation of the nonlocal nature in the propagation dynamics of a cusp-type caustic beam. Figures 2 and 3 clearly exemplify that the self-healing phenomenon in caustic beams is a relative property. These beams then possess a dual behavior: they can behave as either a self-healing beam or a self-breaking one.

On the other hand, what happens if the obstacle is symmetrically placed at the right lateral side instead of the left one? As a cusp-type beam is a beam that is symmetric in its intensity distribution, the beam has identical propagation dynamics but specularly inverted, i.e., the caustic hole takes place on the left caustic curve. In return, a fold-type beam is asymmetric in intensity distribution such that opposite obstruction will induce different propagation dynamics. The obstacle is now located in the dark region of the fold-type beam. It was verified (not depicted) that the $\mathrm{AB}$ remains unaltered in this framework following the dynamics depicted in Fig. 1( $\left.a_{1}\right)$. No ray exists in this region for fold-type beams since the shadow region is only constituted by an evanescent field that does not contribute to the wave-field formation. The above matters open a way to experimentally distinguish between fold-type and cusp-type optical beams: putting an obstacle in a shadow region. If a breaking in the intensity distribution of the beam occurs, the beam is a cusp-type one. Otherwise, the beam is a fold-type one.

\section{EXPERIMENTAL RESULTS}

We carry out the experimental realization of the propagation schemes analyzed above in order to support the theoretical predictions. The beam complex field amplitude $U$ given by Eq. (7) was encoded as a phase-only computer-generated hologram $(\mathrm{CGH})$ following the approach reported in [15,29]. In our case, the $\mathrm{CGH}$ was addressed into a programmable reflective LCoS-SLM (Holoeye LETO, 8-bit 256 gray-levels, pixel pitch of $6.4 \mu \mathrm{m}$ and $1920 \times 1080$ pixels) calibrated for a $2 \pi$ phase shift at the wavelength $\lambda=532 \mathrm{~nm}$ and corrected from static aberrations, as reported in [30]. In order to generate the one-dimensional $\mathrm{AB}$ and $\mathrm{SAB}$, the hologram was illuminated by a collimated laser beam $(\lambda=532 \mathrm{~nm})$ and then focused by a converging cylindrical lens (N-BK7 glass, $f=80 \mathrm{~cm}$ ) that was placed at a distance $f$ in front of the spatial light modulator (SLM) phase array. The origin $z=0$ coincides with the focal plane position of the focusing lens. The obstacle was made by depositing an ink line on 

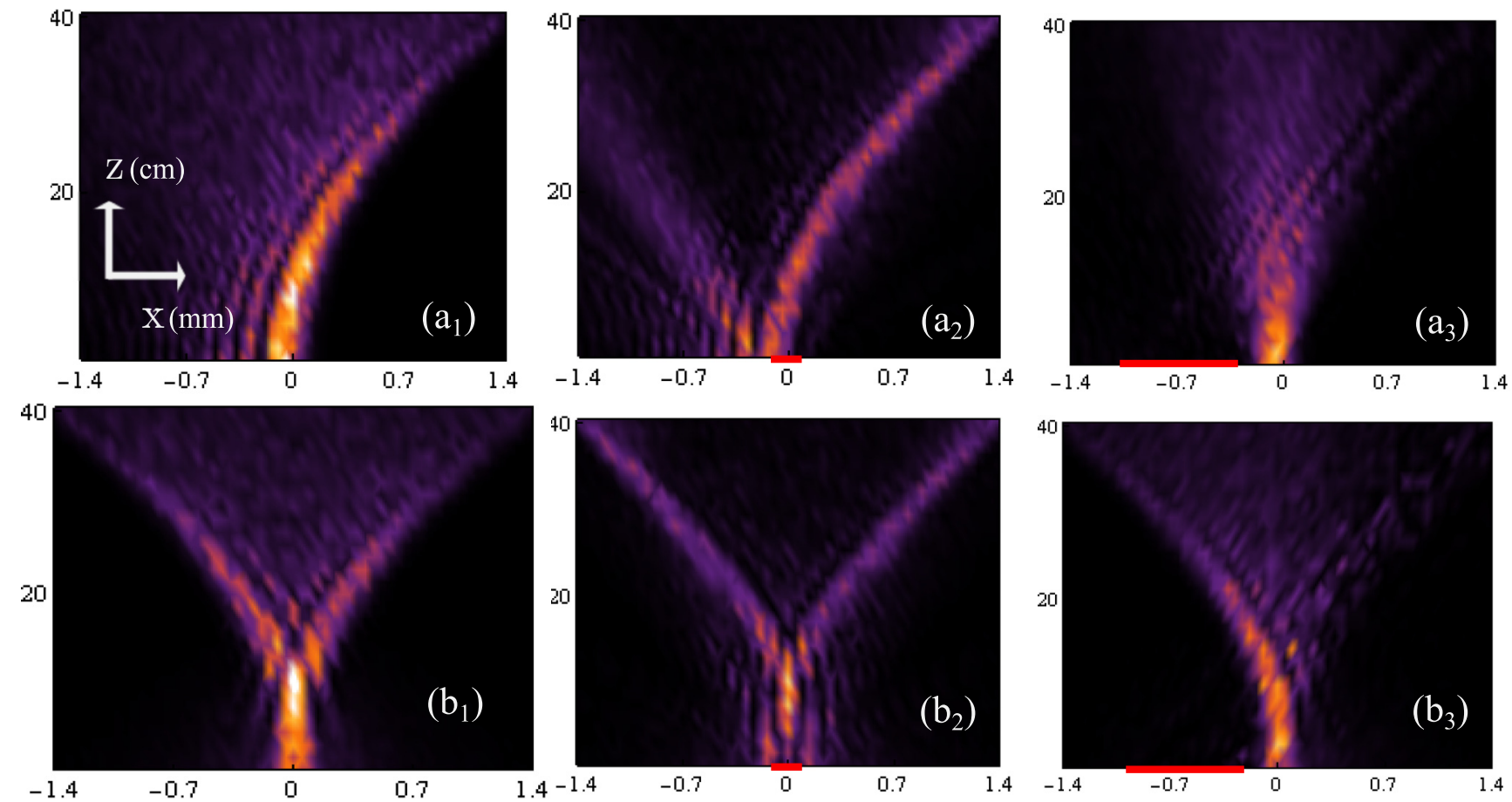

FIG. 4. Experimental normalized intensity distribution $\left(I / I_{\max }\right)$, in terms of the transverse and longitudinal spatial coordinates, $x$ and $z$, from the Fourier plane $(z=0)$ for (a) AB representing a fold-caustic beam and (b) SAB representing a cusp-caustic beam. $\left(a_{1}\right),\left(b_{1}\right)$ Obstacle-free propagation. $\left(a_{2}\right),\left(b_{2}\right)$ Propagation in the presence of an obstacle situated at the beam center. $\left(a_{3}\right),\left(b_{3}\right)$ Propagation in the presence of an obstacle blocking the left lateral side of the beams. The red (thick) segment on the Fourier plane indicates the location and size of the obstacle.

a glass cover slide placed at the Fourier plane (length of $0.2 \mathrm{~mm}$ for the central obstacle and $0.7 \mathrm{~mm}$ for the lateral obstacle). The propagation dynamics of these beams were then recorded as a function of propagation distance for $z>0$ by translating the imaging apparatus, i.e., an sCMOS camera (Hamamatsu, Orca Flash 4.0, 16-bit gray-level, pixel size of $6.5 \mu \mathrm{m})$. Specifically, 200 intensity patterns were measured in the range $z \in[0,40] \mathrm{cm}$ beginning from the Fourier plane. The resulting intensity patterns for $\mathrm{AB}$ and for $\mathrm{SAB}$ are displayed in Figs. 4(a) and 4(b), respectively. Figures $4\left(a_{1}\right)$ and $4\left(b_{1}\right)$ show the results for the obstacle-free propagation that agree with the simulations in Figs. $1\left(a_{1}\right)$ and $1\left(b_{1}\right)$. Figures $4\left(a_{2}\right)$ and $4\left(b_{2}\right)$ show the beam propagation when the obstacle is located at the beam center on the optical axis. For the fold-type beam [Fig. 4( $\left.a_{2}\right)$ ], it is observed that the main lobe is not broken. There only exists a marked depletion of the secondary lobes about the optical axis. For the cusp-type beam case, an appreciable interference is observed around the central lobe immediately behind the obstruction, although no breaking of the specular curved lobes is produced during propagation. These results demonstrate a clear self-healing process for both types of caustic beams, in full accordance with the intensity patterns presented in Figs. 2( $\left.a_{1}\right)$ and 2( $\left.b_{2}\right)$. Finally, Figs. 4( $\left.a_{3}\right)$ and $4\left(b_{3}\right)$ show the beam propagation when the obstruction is placed at the left sideways of the beams. For the fold-type beam [Fig. 4(a $\left.a_{3}\right)$, a rupture on the main curved lobe is observed in the opposite sideways, such that the beam has no resemblance to the original obstacle-free beam beyond $z=0$. For the cusp-type beam, a rupture on the right lobe is also observed while the left lobe remains unaltered. The resultant beam has no likeness with the original obstacle-free beam of Fig. 1( $\left.b_{1}\right)$. These results demonstrate a clear self-breaking process for both types of caustic beams, in full agreement with the theoretical intensity patterns presented in Figs. 3( $\left.\mathrm{a}_{1}\right)$ and $3\left(b_{2}\right)$. Besides, we have also performed the experiments by changing the obstruction to the right sideways. The obtained results (not shown in Fig. 4) confirm the full immutability for the $\mathrm{AB}$ with the obstruction located in the absolute dark region. In return, for the $\mathrm{SAB}$, the specular symmetric behavior is confirmed. The left lobe is depleted on account of the obstruction placed at the right side, which is the specular counterpart of the process shown in Fig. 4( $\left.b_{3}\right)$. In summary, it was demonstrated that fold-type and cusp-type optical beams present a dual behavior depending on the obstruction location.

\section{FINAL REMARKS AND CONCLUSIONS}

A full analysis of both fold-type and cusp-type caustic beams under different setups of obstructions was theoretically and experimentally performed. In order to establish the overall features, we have used two complementary approaches: first, the caustic curve formation from rays and caustic rupture due to obstruction under the framework of catastrophe theory; second, the wave-field analysis using the angular spectrum and Huygens-Fresnel representations. The theoretical results were supported by means of experiments. It was confirmed that the self-healing property of caustic optical beams is a quite relative property. In fact, fold-type and cusp-type beams behave as self-healing beams by blocking the main intensity peak. In parallel, these beams can also behave as self-breaking 
ones. In fact, a counterintuitive phenomenon is revealed: By blocking a sideways of the beam without touching the main intensity peak, the curved lobe at the opposite side breaks down. The regeneration process as well as the rupture one are due to a strong nonlocal propagation dynamics that caustic beams follow, which is distinguishable from the conventional beams. The process of formation of the caustic curve for both kinds of beams is due to side ray contributions. The main intensity lobe cannot be viewed as a local generation of itself, as was clearly demonstrated by the ray scheme. In spite of the above-mentioned similar behaviors between fold-type and cusp-type facing obstructions, deep differences were confirmed among both beams too. The fold-caustic beam remains unaltered facing the obstacle located in the dark region outside the caustic region, while the cusp-caustic beam is broken under the same conditions. This fact points out the presence of a propagating wave field in addition to the evanescent one in the outside region to the caustic curve. The breaking of the cusp beam makes evident the key role of this propagating field.
This study provides an insight into the knowledge of the unusual dynamics mechanisms in fold-type and cusp-type caustic optical beams. The results obtained here cast down the established idea that the Airy beam is necessarily a self-healing one since any caustic beam behaves in a dual manner, i.e., they can self-break by blocking a dark region. These facts open up different perspectives for the applications in which the self-healing properties of the beam are relevant.

\section{ACKNOWLEDGMENTS}

Financial support is acknowledged from Consejo Nacional de Investigaciones Científicas y Técnicas (CONICET-Argentina) under Project No. PIP 2015-1017 11220150100435CO, and Spanish Ministerio de Economía y Competitividad (MINECO) under Project No. TEC201457394-P. P.V. acknowledges support from the Conselho Nacional de Desenvolvimento Científico e Tecnológico (CNPq)Brazil. R.L. acknowledges the support from the Anhui Natural Science Foundation (Grant No. 1708085MF143).
[1] G. A. Siviloglou and D. N. Christodoulides, Opt. Lett. 32, 979 (2007).

[2] G. A. Siviloglou, J. Broky, A. Dogariu, and D. N. Christodoulides, Phys. Rev. Lett. 99, 213901 (2007).

[3] J. D. Ring, J. Lindberg, A. Mourka, M. Mazilu, K. Dholakia, and M. R. Dennis, Opt. Express 20, 18955 (2012).

[4] J. Broky, G. A. Siviloglou, A. Dogariu, and D. N. Christodoulides, Opt. Express 16, 12880 (2008).

[5] X. Chu, G. Zhou, and R. Chen, Phys. Rev. A 85, 013815 (2012).

[6] Y. Qian and F. Wyrowski, Optik 125, 3876 (2014).

[7] F. Zhuang, Z. Zhu, J. Margiewicz, and Z. Shi, Opt. Lett. 40, 780 (2015).

[8] L. Zhang, F. Ye, M. Cao, D. Wei, P. Zhang, H. Gao, and F. Li, Opt. Lett. 40, 5066 (2015).

[9] J. Baumgartl, M. Malizu, and K. Dholakia, Nat. Photon. 2, 675 (2008)

[10] Y. Gu and G. Gbur, Opt. Lett. 35, 3456 (2010).

[11] Y. Hu, P. Zhang, C. Lou, S. Huang, J. Xu, and Z. Chen, Opt. Lett. 35, 2260 (2010).

[12] J. Rogel-Salazar, H. A. Jiménez-Romero, and S. Chávez-Cerda, Phys. Rev. A 89, 023807 (2014).

[13] Y. Kaganovsky and E. Heyman, Opt. Express 18, 8440 (2010).

[14] P. Vaveliuk, A. Lencina, J. A. Rodrigo, and Ó. Martínez-Matos, Phys. Rev. A 92, 033850 (2015).

[15] P. Vaveliuk, A. Lencina, J. A. Rodrigo, and Ó. Martínez-Matos, Opt. Lett. 39, 2370 (2014).

[16] P. Vaveliuk, A. Lencina, J. A. Rodrigo, and Ó. Martínez-Matos, J. Opt. Soc. Am. A 32, 443 (2015).
[17] P. A. Quinto-Su and R. Jáuregui, Opt. Express 22, 12283 (2014).

[18] I. Epstein and A. Arie, Phys. Rev. Lett. 112, 023903 (2014).

[19] E. Greenfield, M. Segev, W. Walasik, and O. Raz, Phys. Rev. Lett. 106, 213902 (2011).

[20] L. Froehly, F. Courvoisier, A. Mathis, M. Jacquot, L. Furfaro, R. Giust, P. A. Lacourt, and J. M. Dudley, Opt. Express 19, 16455 (2011).

[21] P. Zhang, Y. Hu, T. Li, D. Cannan, X. Yin, R. Morandotti, Z. Chen, and X. Zhang, Phys. Rev. Lett. 109, 193901 (2012).

[22] Yu. A. Kravtsov and Yu. I. Orlov, Caustics, Catastrophes and Wave Fields, 2nd ed. (Springer-Verlag, Berlin, 1999).

[23] M. V. Berry, in Catastrophe Optics: Morphologies of Caustics and Their Diffraction Patterns, edited by E. Wolf, Progress in Optics XVII (North-Holland, Amsterdam, 1989).

[24] J. F. Nye, Natural Focusing and Fine Structure of Light Caustics and Wave Dislocations (Taylor \& Francis, London, 1999).

[25] J. W. Goodman, Introduction to Fourier Optics (McGraw-Hill, New York, 1968).

[26] P. Vaveliuk and Ó. Martínez-Matos, Opt. Express 20, 26913 (2012).

[27] A. E. Siegman, Lasers (University Science Books, Mill Valley, California, 1986).

[28] Z.-X. Fang, Y.-X. Ren, L. Gong, P. Vaveliuk, Y. Chen, and R.-D. Lu, J. Appl. Phys. 118, 203102 (2015).

[29] J. A. Davis, D. M. Cottrell, J. Campos, M. J. Yzuel, and I. Moreno, Appl. Opt. 38, 5004 (1999).

[30] J. A. Rodrigo, T. Alieva, A. Cámara, Ó. Martínez-Matos, P. Cheben, and M. L. Calvo, Opt. Express 19, 6064 (2011). 